Check for updates

Cite this: RSC Adv., 2019, 9, 5540

Received 6th January 2019

Accepted 5th February 2019

DOI: 10.1039/c9ra00117d

rsc.li/rsc-advances

\section{Quantitative determination of the spring entropy effect and its indication of the conformational change of polymer coils with varying concentration in aqueous poly $(\mathrm{N}$-isopropylamide) solutions $\uparrow$}

\author{
Li Song, Jiaxiang Lin, Panpan Liu, Jingqing Li, (D) Shichun Jiang (D) \\ and Dinghai Huang (D) *
}

\begin{abstract}
The lower critical solution temperature (LCST) phase separation behaviors of thermosensitive poly $(N$ isopropylacrylamide) (PNIPAM) aqueous solutions were investigated by power-compensation differential scanning calorimetry (DSC). The entropic effect and hence the change of swelling state of PNIPAM polymer coils in homogeneous concentrated aqueous solutions with varied solution composition was elucidated by the isothermal enthalpy demixing recovery behaviors in distinct concentration regions.
\end{abstract}

\section{Introduction}

Since thermoresponsive aqueous poly( $N$-isopropylacrylamide) (PNIPAM) exhibits a type II lower critical solution temperature (LCST) phase separation behavior ${ }^{1-3}$ close to physiological conditions $\left(T_{\mathrm{c}}=32{ }^{\circ} \mathrm{C}\right)$, it has been utilized in a variety of applications such as drug delivery systems, ${ }^{4,5}$ stimuli-responsive $^{6-8}$ and multi-stimuli responsive ${ }^{9-12}$ materials, separation of suspension solutions, ${ }^{13}$ and separation material in chromatographic procedures ${ }^{14}$ only to name a few. Many reports in the literature indicate that the conformational change of the polymer plays a critical role in the dramatic physical property changes occurring around the LCST in PNIPAM or grafted materials..$^{15-21}$

As one of the fundamental problems in polymer physics, the conformational change of a linear flexible polymer chain from an expanded coil to a collapsed globule has been extensively studied in the past half century. ${ }^{22-28}$ However, most of these works focus on extremely dilute or semidilute solutions, investigations on the change in size of polymer coils in concentrated solution are very limited, especially for aqueous solutions, which have only been conducted in a narrow concentration region. ${ }^{29-31}$

Entropic effect induced by the collapse of polymer coils is much different from that induced by the extension fashion, the latter is usually termed as elastic entropy or entropic elasticity, while the former is termed as spring entropic effect. ${ }^{32,33}$ It has been noted that the theoretical treatment model of spring

Department of Polymer Material Science and Engineering, Institution of Advanced Polymer Materials, School of Material Science and Engineering Tianjin University, Tianjin, 300350, China.E-mail: dhuang@tju.edu.cn

$\dagger$ Electronic supplementary information (ESI) available. See DOI: 10.1039/c9ra00117d entropy is much different from that of elastic entropy ${ }^{33,34}$ The elastic entropy in macroscopic or microscopic chain extension fashion has been investigated thoroughly, ${ }^{35-38}$ however, the spring entropy in the collapse mode is rarely addressed.

As discussed in a recent work, ${ }^{39}$ in the demixing process of a polymer solution, the heat or enthalpy $\left(\Delta H_{\text {total }}\right)$ and free energy $\left(\Delta G_{\text {total }}\right)$ of demixing and the total demixing entropy $\left(\Delta S_{\text {total }}\right)$ can be described as:

$$
\begin{aligned}
\Delta G_{\text {total }} & =\Delta H_{\text {total }}-T \Delta S_{\text {total }}=\Delta H_{\text {total }} \\
& -T\left(\Delta S_{\text {conf }}^{\mathrm{p}}+\Delta S_{\text {solvent }}+\Delta S_{\mathrm{r}-\mathrm{t}}+\Delta S_{\text {other }}\right)
\end{aligned}
$$

where $\Delta S_{\text {solvent }}$ is the entropy of solvent water, $\Delta S_{\text {conf }}^{\mathrm{p}}$ is the change of the conformational entropy and $\Delta S_{\mathrm{r}-\mathrm{t}}$ is the rotational-translation entropy of polymer, $\Delta S_{\text {other }}$ is the contributions to entropy from other unrecognized sources.

As proposed by Budkov et al., ${ }^{40}$ in the framework of the mean-field approximation, the Gibbs free energy of polymer coils takes the form:

$$
\begin{aligned}
\Delta G_{\mathrm{p}}\left(R_{\mathrm{g}}\right)= & \Delta H_{\mathrm{p}}-T \Delta S_{\mathrm{p}} \\
= & K_{\mathrm{B}} T\left(\frac{9}{4}\left(\alpha^{2}+\frac{1}{\alpha^{2}}\right)-\frac{3}{2} \ln \alpha^{2}\right) \\
& -N K_{\mathrm{B}} T \ln \left(1-\frac{N v_{\mathrm{m}}}{V_{\mathrm{g}}}\right)-\frac{a_{\mathrm{p}} N^{2}}{V_{\mathrm{g}}}
\end{aligned}
$$

where $\alpha$ is the chain expansion factor, $\alpha=\left\langle R_{\mathrm{g}}\right\rangle /\left\langle R_{\mathrm{g}}\right\rangle_{0}, K_{\mathrm{B}}$ is the Boltzmann constant, $v_{\mathrm{m}}=2 \pi \sigma_{\mathrm{m}}{ }^{3} / 3$ is the van der Waals volume of the monomers $\left(\sigma_{\mathrm{m}}=\sigma_{\mathrm{mm}}\right.$ denotes the monomer's effective diameter), and $V_{\mathrm{g}}=4 \pi{R_{\mathrm{g}}}^{3} / 3$ is the gyration volume of the polymer coils.

According to eqn (2), when a polymer solution is subjected to phase separation from two extremely conditions under the same temperature change procedure, that is, one phase 
separated from swelling coils and the other separated from collapsing globules, since the enthalpic effect due to the change of polymer-solvent interaction in the process of phase separation is almost the same, the influence of polymer coil dimensions will mainly correlated with the entropic effect.

In our recent work ${ }^{\mathbf{4 1}}$ we showed that in concentrated aqueous PNIPAM solutions, phase separation from different swelling states can be achieved isothermally at a lower temperature than the LCST for different times after subjecting the first phase separation to a heating scan, and the entropic effect due to the change of swelling state was qualitatively discussed. Subsequently, similar entropic effects were also observed in another type III LCST behavior poly(vinyl methyl ether) (PVME) aqueous solutions. ${ }^{39,42}$ In contrast to the complicate chemical structure in PNIPAM, the structure of PVME is very simple and there is only one polar oxygen ether atom in each repeating unit and can form hydrogen bond with a maximum of two water molecules. The DSC results showed that below the critical water concentration of $38.3 \mathrm{wt} \%$ (i.e. 1 : 2 mole ratio of PVME repeating unit and water), the time independent of demixing enthalpy recovery behavior indicates that the PVME coil is in a globular state. A typical sigmoidal recovery behavior of demixing enthalpy above $38.3 \mathrm{wt} \%$ is ascribed as the reswelling of the collapsed polymer coils induced by the entropic effect. The increased difference between the upper and lower limits indicates the continued swelling of the PVME coils. Above $65 \mathrm{wt} \%$, a dominant diluting effect can be observed, and a much longer phase separation time is needed to reach the expected lower limit. ${ }^{39}$

Although the swelling state of polymer coils in extremely dilute solution and rather compact globule state of polymer chains in bulk state are clear for PNIPAM, it is still extremely challenging and difficult to confirm when and how the compact PNIPAM globules begin to swell with the continuous dilution of water solvent in concentrated solutions. Based on the above studies, ${ }^{\mathbf{3 9 , 4 1 , 4 2}}$ in this work, the entropic effects in the demixing enthalpy recovery behavior in different concentration regions and its implications on the conformational changes of polymer coils swelling state in aqueous PNIPAM solutions are investigated.

Moreover, it should be noted that in the previous investigations the determination of entropic contributions in thermodynamic energy term is usually elucidated out from a series of molecules with similar chemical or complex characters, ${ }^{43-47}$ in this study and our recent work about PVME aqueous solutions ${ }^{39}$ we showed that the entropic effect can be directly determined from a single polymer solution.

\section{Experimental}

\subsection{Sample preparation}

Poly( $N$-isopropylacrylamide) (PNIPAM) was obtained from Aldrich Chemical Company Inc. Its mass average molecular weight $\left(M_{\mathrm{w}}\right)$ characterized by gel permeation chromatography (GPC) is $3.59 \times 10^{4} \mathrm{~g} \mathrm{~mol}^{-1}$ and $M_{\mathrm{w}} / M_{\mathrm{n}}=1.51$. The aqueous solution sample was dried in a vacuum oven at $65{ }^{\circ} \mathrm{C}$ with sufficient $\mathrm{P}_{2} \mathrm{O}_{5}$ powder as the drying agent for a few days until the weight of the sample stopped changing (error within
$0.01 \mathrm{wt} \%$ ) to obtain pure PNIPAM sample. The water used in this work was deionized and doubly distilled.

\subsection{DSC experiments}

A series of PNIPAM aqueous solutions with various PNIPAM content were prepared from the dried PNIPAM sample by adding appropriate amounts of deionized water in aluminum DSC crucible pans. The amount of added water is slightly higher than the anticipated composition of prepared solution, and excess water was simply removed by natural evaporation in open air for an appropriate period of time to the desired solution concentration, the pan was then hermetically sealed quickly. The prepared aqueous solution samples were left undisturbed for at least 7 days at room temperature and without any weight loss to ensure a good sealing effect before any DSC measurements.

The DSC measurements were performed on a powercompensation Perkin Elmer Diamond DSC with a Freon intraCooler $2 \mathrm{P}$ cooling accessory (the cooling agent can be cooled to as low as $-95^{\circ} \mathrm{C}$ ). The power-compensation DSC was selected in this investigation because its furnace has low mass and small dimensions, ensuring a much faster heat transfer than heat-flux calorimeters. The small gap between the furnace and the cooling system, which is reduced by metallic guard ring inserts, promotes effective cooling.

All measurements were made in nitrogen atmosphere. The DSC temperature was calibrated using a two-point method with high-purity indium and mercury at a heating rate of $10{ }^{\circ} \mathrm{C} \mathrm{min}^{-1}$. The heat flow was calibrated with a standard sapphire sample. The sample mass varied from 5.0 to $12.5 \mathrm{mg}$.

For the samples with water concentrations lower than $65 \mathrm{wt} \%$, the aqueous PNIPAM solution samples were heated from $15{ }^{\circ} \mathrm{C}$ to $55{ }^{\circ} \mathrm{C}$ at a heating rate of $10{ }^{\circ} \mathrm{C} \min ^{-1}$ and then isothermally at $55{ }^{\circ} \mathrm{C}$ for $1 \mathrm{~min}$ to ensure that the samples completely undergo phase separation process. Consequently, the samples were cooled from $55^{\circ} \mathrm{C}$ to $15^{\circ} \mathrm{C}$ with a relatively fast cooling rate of $50{ }^{\circ} \mathrm{C} \mathrm{min}^{-1}$; after being kept isothermally at $15{ }^{\circ} \mathrm{C}$ for different times varied from $0.1 \mathrm{~min}$ to up to $10^{4} \mathrm{~min}$, the samples were heated to $55{ }^{\circ} \mathrm{C}$ at $10{ }^{\circ} \mathrm{C} \mathrm{min}{ }^{-1}$ for a second time, and a series of DSC heat flow curves with different isothermal recovery times at $15{ }^{\circ} \mathrm{C}$ could be obtained. When the water concentration is equal or higher than $65 \mathrm{wt} \%$, the isothermal phase separation time at $55{ }^{\circ} \mathrm{C}$ was increased as indicated for different samples. The other cooling condition and isothermal time at $15{ }^{\circ} \mathrm{C}$ are the same as those for samples with concentrations lower than $65 \mathrm{wt} \%$.

\section{Results and discussion}

\subsection{Distinctive time dependency of demixing enthalpy recovery in different concentration solutions}

Fig. 1 showed the phase separation peaks in DSC curves collected from the second heating scan, which indicated different changes with isothermal recovery time at $15{ }^{\circ} \mathrm{C}$ for PNIPAM aqueous solution with $45 \mathrm{wt} \%$ (a) and $60 \mathrm{wt} \%$ (b) water content. As shown in Fig. 1(a), the heat flow curves of 


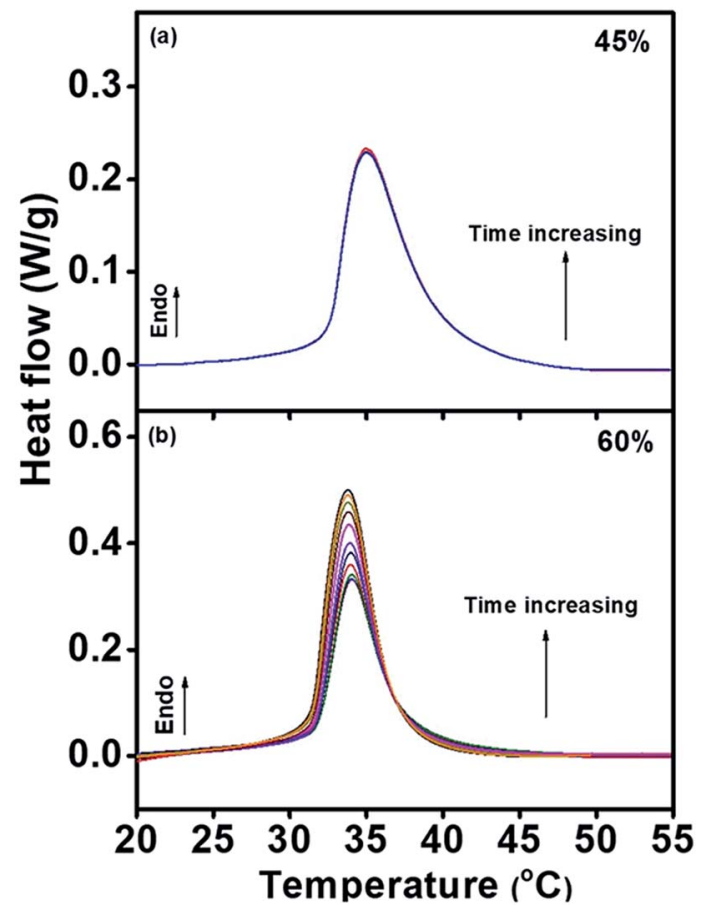

Fig. 1 The DSC curves of PNIPAM aqueous solution with (a) 45 wt\% and (b) 60 wt\% water content in the second heating scan after phase separation from equilibrium state as the increase of isothermal time at $15^{\circ} \mathrm{C}$.

endothermic phase separation peak demonstrate no change with the increase of isothermal time. However, in Fig. 1(b) the heat flow curves significantly increase with the raising of isothermal time. These results prove that with the rising of isothermal time, heat flow of endothermic phase separation peak is unchanged for PNIPAM aqueous solutions with low water content, and on the contrary, remarkable change of heat flow occurs with the increase of isothermal time in PNIPAM aqueous solutions with high water content.

As shown in Fig. 2, the integrated demixing enthalpy remain unchanged with the change of logarithmic isothermal time for PNIPAM aqueous solution with $45 \mathrm{wt} \%$ water content, while with the increasing of logarithmic isothermal time, demixing enthalpy firstly increases and then maintain constant for the sample with $60 \mathrm{wt} \%$ water content. When the isothermal time is less than $3 \mathrm{~min}$, the demixing enthalpy retain almost constant $\left(\sim 8.5 \mathrm{~J} \mathrm{~g}^{-1}\right)$ over time. If the isothermal time situates between $3 \mathrm{~min}$ and $120 \mathrm{~min}$, the demixing enthalpy will observably increase with the raising of isothermal time. In addition, the demixing enthalpy finally plateaus at about $13 \mathrm{~J} \mathrm{~g}^{-1}$ when the isothermal time is $120 \mathrm{~min}$ or even longer.

For the convenience of later discussions, the concept of the lower limit (isothermal recovering time at $15{ }^{\circ} \mathrm{C}$ for less than 1 $\mathrm{min}$ ) and upper limit of demixing enthalpy (isothermal recovering time at $15{ }^{\circ} \mathrm{C}$ for longer than $120 \mathrm{~min}$ ) is put forward.

As discussed in the recent work about PVME aqueous solutions, ${ }^{39}$ the distinctive demixing enthalpy recovery behaviors in different concentration solutions may be attributed to the entropic effect due to changes in polymer chain swelling states.

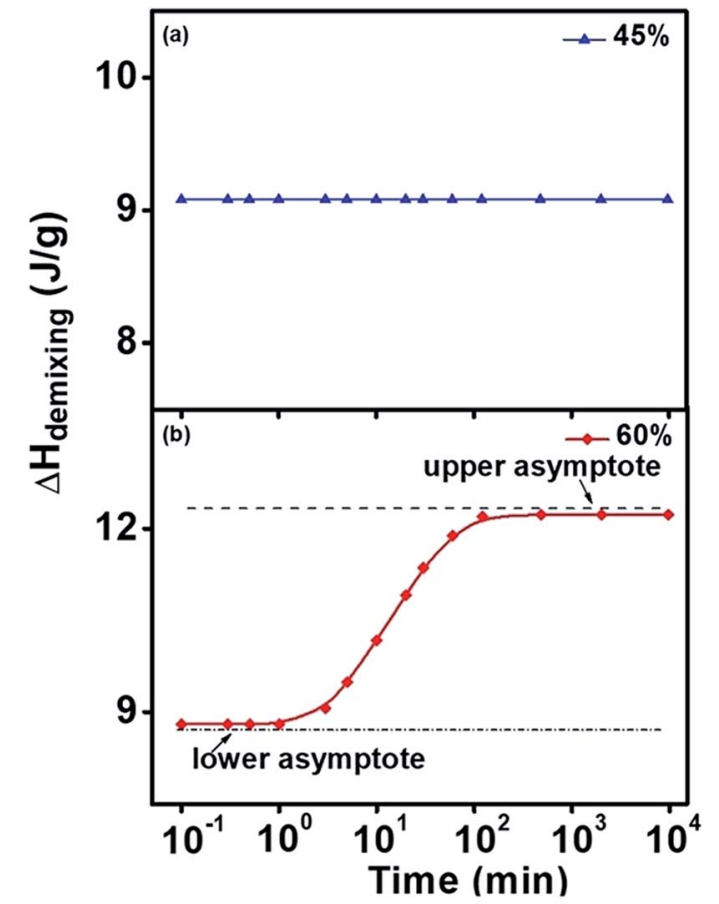

Fig. 2 The demixing enthalpy curves with various isothermal time in (a) $45 \mathrm{wt} \%$ and (b) $60 \mathrm{wt} \%$ water content of PNIPAM aqueous solution.

It is well known that the Gibbs free energy and entropy of polymer coils is correlated with the chain expansion factor. ${ }^{34,40}$ In a polymer solution, the polymer chain in a swelling coil state is similar to a stretched spring, while that in a relatively dense globule state is similar to a compressed spring. If the coil-toglobule transition process in the polymer solution is fast enough, the spring entropic effect caused by the change in the swelling states of the polymer chains could not be ignored and it is possible to be detected by the DSC method. That is probably the underlying reason for the distinct characteristics of demixing enthalpy recovery behaviors that observed in different concentration regions.

\subsection{Concentration influence with the recovery time dependency}

As reported by our previous work, ${ }^{41}$ the time dependence of demixing enthalpy recovery behavior showed distinct features in four concentration regions: (I) $0 \mathrm{wt} \%<W_{\text {water }} \leq 24 \mathrm{wt} \%$ (water concentration, similarly hereinafter), (II) $24 \mathrm{wt} \%<W_{\text {water }}$ $\leq 49$ wt $\%$, (III) $49 \mathrm{wt} \%<W_{\text {water }}<65 \mathrm{wt} \%$, (IV) $W_{\text {water }} \geq 65 \mathrm{wt} \%$. When the water concentration is equal or lower than $24 \mathrm{wt} \%$, only a glass transition behavior exists and no sign of phase separation can be observed for the mixtures ${ }^{\mathbf{4 1}}$ (The DSC curves is showed in Fig. S1 in ESI $\dagger$ ). Thus, in this work, only the other three concentration regions involved LCST phase separation behaviors will be discussed.

As shown in Fig. 3, the demixing enthalpy of three samples with different water concentrations remained almost unchanged with increasing isothermal time, indicating the time independent behavior. The result is obviously different 


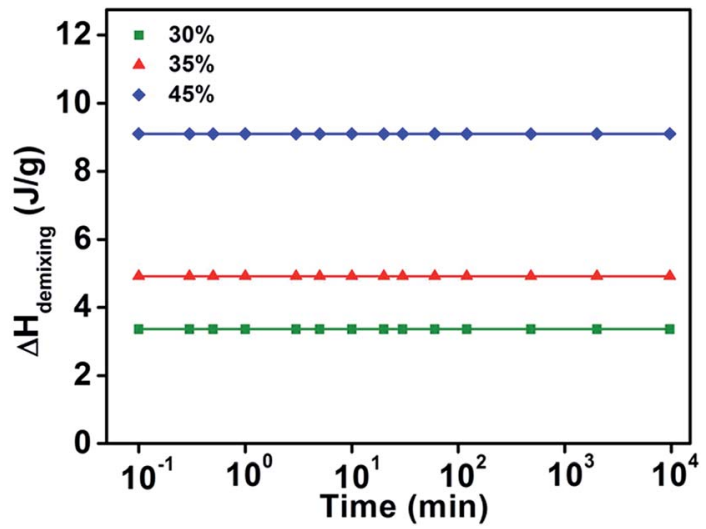

Fig. 3 The change of integrated demixing enthalpy of PNIPAM aqueous solution versus logarithmic isothermal time under $15^{\circ} \mathrm{C}$ in region II (24-49 wt\% water concentration).

from the demixing enthalpy recovery behavior as observed in the PNIPAM solution with $60 \mathrm{wt} \%$ water concentrations. In addition, the measured demixing enthalpy increases with the increasing of water content in this concentration region. The isothermal time independent of demixing enthalpy behavior illustrated in Fig. 3 indicates that the enthalpy is only contributed by the deterioration of hydrogen bond in the molecular complex formed between PNIPAM and water, according to the similar results observed in PVME aqueous solutions. ${ }^{39}$

When the water concentration is between $49 \mathrm{wt} \%$ and $65 \mathrm{wt} \%$, the typical recovery behavior of demixing enthalpy can be found. Moreover, in the case of short isothermal time, the lower limit of demixing enthalpy decreases with raising of water concentration, while the upper limit of demixing enthalpy corresponding to longer isothermal time increase with the rising of water concentration in region III. For three samples with different water concentrations, with the increasing of logarithmic isothermal time, their demixing enthalpies all firstly increase and then remain constant in a sigmoidal manner.

The typical sigmoidal shape of isothermal time dependency of the demixing enthalpy recovery behavior in Fig. 4 indicates

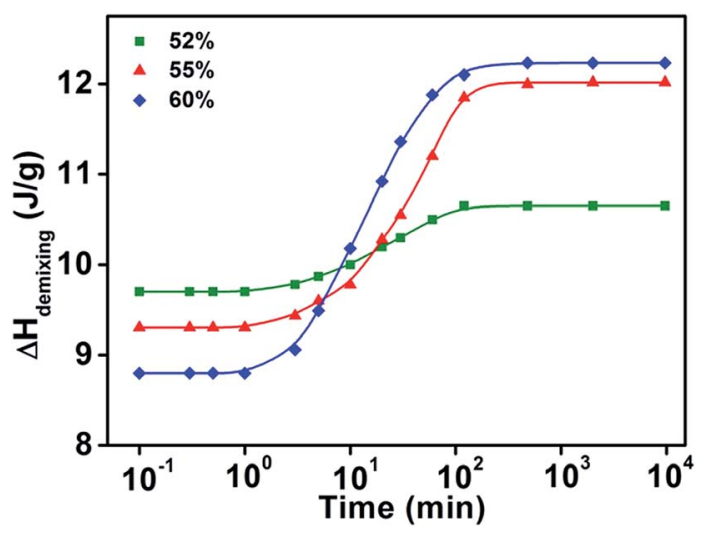

Fig. 4 The change of integrated demixing enthalpy of aqueous PVME solution versus logarithmic isothermal time under $15{ }^{\circ} \mathrm{C}$ in region III (49-65 wt\% water concentration). that the enthalpy recovery behavior of PNIPAM aqueous solution is influenced by two distinct factors. The first transition, which appeared at approximately $3 \mathrm{~min}$, presumably to be dominated by the penetration of water back into the phaseseparated and aggregated polymer phase when the temperature dropped to below the LCST; and the second transition at approximately $120 \mathrm{~min}$ may be dominated by the relaxation process of whole polymer chains from a compact collapsed globular state to a swelling coil state. Moreover, the later recovery time is in agreement with the experimentally determined relaxation time scale of a whole polymer chain. ${ }^{\mathbf{4 8 , 4 9}}$

When the water concentration is lower than $65 \mathrm{wt} \%$, isothermal phase separation at $55{ }^{\circ} \mathrm{C}$ for $1 \mathrm{~min}$ is enough for PNIPAM solutions to reach a fully collapsed state, as shown in Fig. 4. However, when the water concentration exceeds $65 \mathrm{wt} \%$, the required isothermal phase separation time at $55{ }^{\circ} \mathrm{C}$ significantly increases with rising water concentration. Furthermore, the detected demixing enthalpy after a short isothermal phase separation time keeps deviating to a higher value with rising water concentration, as shown by the open symbols in Fig. 5(a). In this plot, the closed symbol and the upper dashed line represent the demixing enthalpy value measured from the polymer solution in an equilibrium state, in other words, the upper limit value is measured after being isothermal at $15^{\circ} \mathrm{C}$ for a sufficiently long time at each concentration indicated in Fig. 5(a).

With the increasing time of isothermal phase separation at $55{ }^{\circ} \mathrm{C}$, the detected demixing enthalpy decreased in a sigmoidal manner versus the logarithmic time. This trend is very similar to that observed in PVME aqueous solutions. ${ }^{39}$ However, as the molecular structure of PNIPAM is much more complicated, the predicted lower limit demixing enthalpy value corresponding to the fully collapsed state as in PVME solutions cannot be obtained.

Similarly, the $90 \mathrm{wt} \%$ water PNIPAM aqueous solution showed continuing increase of phase separation time at $55^{\circ} \mathrm{C}$, making it possible to detect the decrease in demixing enthalpy by DSC method, as illustrated in Fig. 6 .

The results observed in Fig. 5(a) and 6 can be interpreted as the influence of aggregation on the retardation of the reswelling process. ${ }^{39}$ As the water concentration increases, the probability that the collapsed polymer globule is in an isolated single state after phase separation also increases accordingly. The reswelling of isolated single polymer globules is much faster than that of aggregated polymer globules and cannot be detected by the DSC method. When isothermal at phase separation temperature for a longer period of time, an increasing amount of polymer globules aggregated, and the tendency for demixing enthalpy to decline became more obvious. Furthermore, the results for the $90 \mathrm{wt} \%$ water solution reveal that with increasing water concentration, the polymer globules are relatively stable even under the phase-separation temperature. Another plausible interpretation to the change in demixing enthalpy with concentration is that in a semidilute or dilute aqueous solution, the polymer chain will take the form of a much looser globule than in a concentrated solution. In fact, $\mathrm{Wu}$ and Wang ${ }^{50}$ estimated that the single-chain globules of PNIPAM in an extremely 

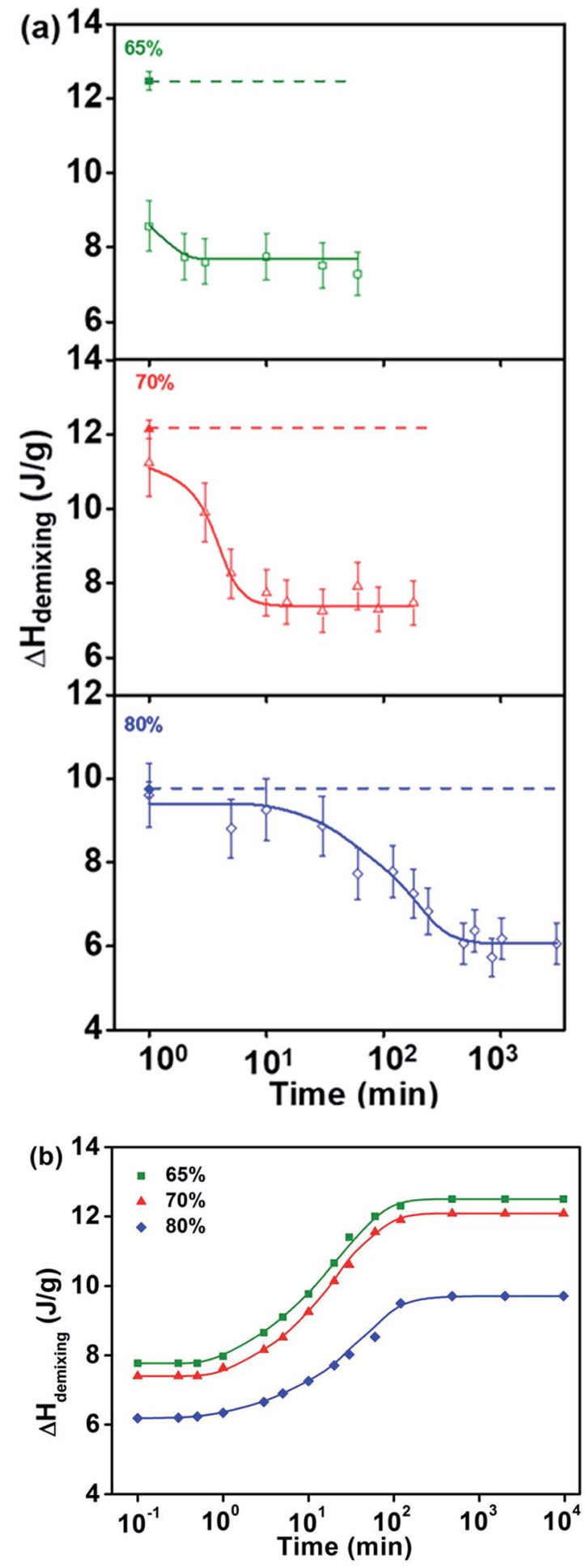

Fig. 5 (a) The change of demixing enthalpy of lower limit (isothermal recovering time of $0.1 \mathrm{~min}$ at $15{ }^{\circ} \mathrm{C}$ ) versus logarithmic of phase separation time at $55^{\circ} \mathrm{C}$ for $65 \mathrm{wt} \%, 70 \mathrm{wt} \%$, and $80 \mathrm{wt} \%$ water PNIPAM aqueous solutions (open symbols). The closed symbols and the upper dashed lines represent the demixing enthalpy measured from well swelling solution samples, that is, the solutions remain undisturbed more than 7 days at ambient temperature. (b) The change of demixing enthalpy of PNIPAM aqueous solution versus different logarithmic isothermal times at $15{ }^{\circ} \mathrm{C}$ in concentration region IV (65 to $80 \mathrm{wt} \%$ water).

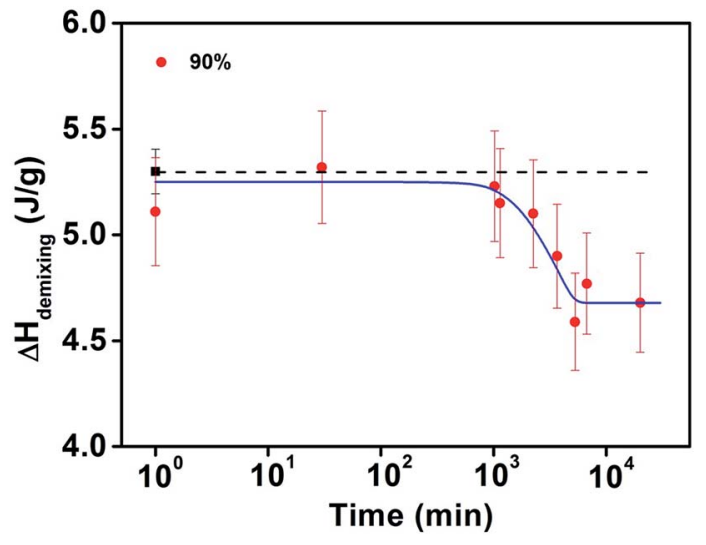

Fig. 6 The change of demixing enthalpy of lower limit (isothermal recovering time of $0.1 \mathrm{~min}$ at $15{ }^{\circ} \mathrm{C}$ ) versus logarithmic of phase separation time at $55^{\circ} \mathrm{C}$ for $90 \mathrm{wt} \%$ water PNIPAM aqueous solution.

dilution aqueous solution above the LCST still contain approximately $60 \%$ water inside its hydrodynamic volume, as probed by a dynamic light-scattering method.

It is worth mentioning that in the PNIPAM aqueous solutions the required isothermal phase separation time for the demixing enthalpy to reach a lower limit is much longer than that in PVME solution in the same concentration when water concentration is $65 \mathrm{wt} \%$ and above ${ }^{39}$ which suggests that the phase separated PNIPAM is more stable in water than PVME.

When the water concentration exceeds $65 \mathrm{wt} \%$, the lower limit of demixing enthalpy corresponding to short isothermal time continues to decrease with increasing water concentration. In contrast, the upper limit of demixing enthalpy ceases to increase and declines with increasing water concentration in region IV compared to region III, but the time dependence of the recovery characteristics are almost the same, as shown in Fig. 5(b). This phenomenon demonstrates that the recovering swelling behavior of the collapsed polymer globules may be controlled by the same factors and is independent of the concentration of the aqueous solution.

Similar phenomenon is also observed in PVME aqueous solution, ${ }^{39}$ and it is very surprising to find that the entanglement effect which is prevailing in polymer solutions cannot be observed in the recovering swelling process after LCST phase separation. The possible factors have been discussed in the previous work, ${ }^{39}$ although the phenomenon of concentration independence of demixing enthalpy recovering behavior is not yet fully understood, these results indicate that the polymer coil collapse process is significantly different from that of shearing elongation mode as measured by viscosity method.

\subsection{The quantitative measurement of the spring entropic effect in phase separation of PNIPAM aqueous solution}

As shown in Fig. 7, the composition dependence of the upper limit of demixing enthalpy is nearly symmetrical and the maximum demixing enthalpy (about $12.46 \mathrm{~J} \mathrm{~g}^{-1}$ ) appears at the $65 \mathrm{wt} \%$ water concentration, which is in good agreement with the results reported in literature.$^{51}$ Moreover, the lower limit of 


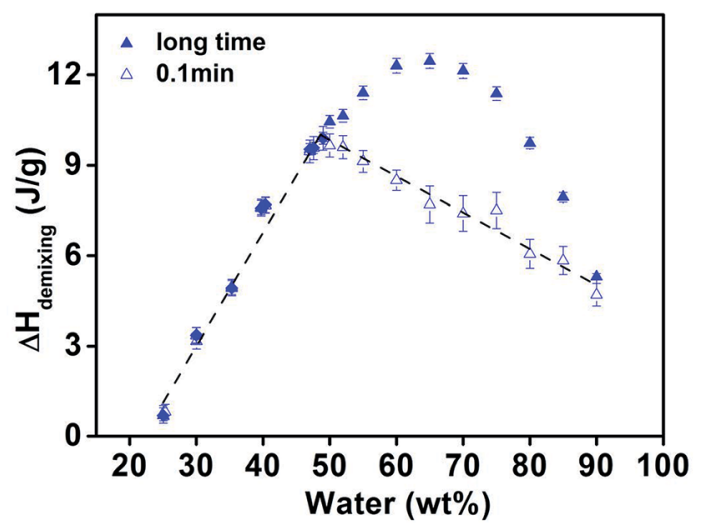

Fig. 7 The change of measured demixing enthalpy for both upper limit (close triangles) and lower limit (open triangles) versus water concentration of PNIPAM aqueous solution. The linear dashed line of the lower limit demixing enthalpy is only drawn as a guide to the eye.

demixing enthalpy observed in the study is also plotted in this figure as open symbols. As illustrated in Fig. 7, the measured lower limit of demixing enthalpy shows no discernible difference from the upper limit value in concentration region II (24$49 \mathrm{wt} \%$ water), and it is almost linear with the change of water content. The increasing of demixing enthalpy with rising water content indicates that the keep contribution of hydrogen bond interaction to form complex between PNIPAM and water in this concentration region.

When the concentration of water is increased to above $49 \mathrm{wt} \%$, the upper limit of demixing enthalpy corresponding to longer isothermal time continues to heighten with the rising of water concentration in region III. However, the lower limit value corresponding to short isothermal time begins to decrease in proportion with the further increase of water concentration above $49 \mathrm{wt} \%$. As discussed in previous study in PVME aqueous solution, ${ }^{39}$ this phenomenon indicates that water molecule is in excess in the solution, the additional water will serve as free solvent and swell the PNIPAM coils. The continuous swelling of polymer coils contributed more entropic effect to the demixing enthalpy, thus the upper limit value still increases with the rising water concentration. While the lower limit value corresponding to short isothermal time that mainly contributed by the phase separation of polymer from fully collapsed state, thus only energetic enthalpic effect can be detected, the hydrogen bonds between PNIPAM and water are already saturated and the excess water mainly serve as diluents solvent, so the lower limit demixing enthalpy is found to keep decreasing with the rising water concentrations.

When the water concentration exceeds $65 \mathrm{wt} \%$, the upper limit of demixing enthalpy ceases to increase and declines with increasing water concentration in region IV in contrast to region III, while the lower limit of demixing enthalpy corresponding to short isothermal time continues to decrease with increasing water concentration. This phenomenon indicates the swelling of polymer coils almost reach their maximum and further added water mainly serves as dilute solution in concentration region IV, which leads to the decline of both upper and lower limits of demixing enthalpy with increasing water concentration.

However, as the chemical structure of PNIPAM is very complicate, the physical meaning of the critical concentrations between the different concentration regions and the influencing factors in aqueous PNIPAM solution are currently poorly understood.

As showed in Fig. 7, the lower limit of demixing enthalpy $\left(\Delta H_{0}\right)$ corresponding to short isothermal time is ascribed to the dissociation of hydrogen bond between PNIPAM and water in the solution, while the upper limit of demixing enthalpy $\left(\Delta H_{\infty}\right)$ corresponding to longer isothermal time is comprehensive reflection of two kinds of effects: one is the dissociation of the hydrogen bond; the other one is entropic effect induced by the change of swelling states of polymer coils during the phase separation, which is equal to the difference between the upper and lower limits. Taking the above mentioned assumptions, normalizing the demixing enthalpies to the amount of PNIPAM as follow leads to the spring entropy effect:

$$
\Delta S=\left(\Delta H_{\infty}-\Delta H_{0}\right) M_{\mathrm{p}} / W_{\mathrm{p}}
$$

where $M_{\mathrm{p}}$ is the molecular weight of repeating unit of PNIPAM, $W_{\mathrm{p}}$ is the weight fraction of PNIPAM in aqueous solution.

The normalized demixing enthalpy induced by entropy spring effect is much more complex in three distinct regions: (II) 24-49 wt\%, (III) 49-65 wt\%, IV) above $65 \mathrm{wt} \%$, as illustrated in Fig. 8. At lower water concentrations region II $(24 \mathrm{wt} \%<$ $W_{\text {water }} \leq 49 \mathrm{wt} \%$ ), the entropic effect is zero, indicating that the polymer chain is in compact globule state and has almost no spring entropy effect in this region. As water concentration further increases to region III ( $49 \mathrm{wt} \%<W_{\text {water }}<65 \mathrm{wt} \%$ ), the normalized entropic effect obviously enhances with increasing of water content, suggesting the continuous swelling of the polymer coil as the increase of water concentration in this region. At the higher water concentrations region IV ( $W_{\text {water }} \geq$ 65 wt\%), the spring entropy only increases slightly with increasing water content, indicating that the swelling degree of polymer coil reaches nearly its maximum when the water concentration is $65 \mathrm{wt} \%$, and further increase in water

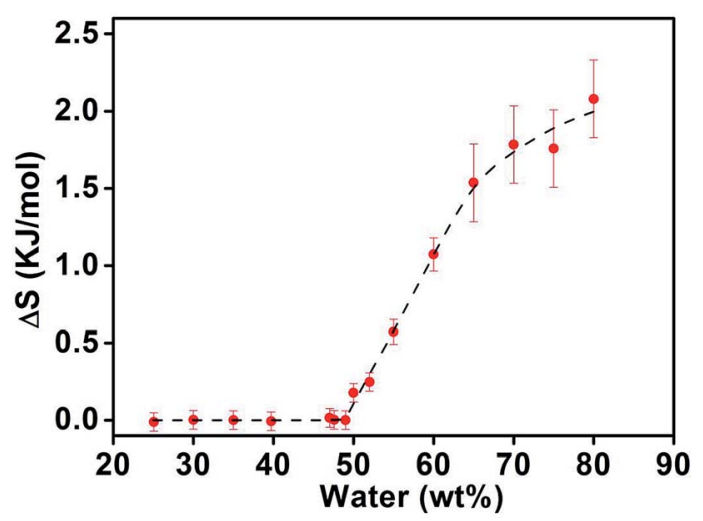

Fig. 8 The change of calculated spring entropy effect versus water concentration of PNIPAM aqueous solution. 
concentration mainly induced dilution effect in the PNIPAM aqueous solution. As the concentration of water increases further to above $80 \mathrm{wt} \%$, the error induced by the convention DSC apparatus will increase significantly, ultrasensitive microcalorimetry (US-DSC) maybe more powerful in the more diluted solutions. $^{52}$

It should be noted that when the water concentration is increase to $80 \mathrm{wt} \%$ and above, as PNIPAM becomes more and more stable in aqueous solution even after phase separation, which can be found from the significantly prolonged phase separation time at $55{ }^{\circ} \mathrm{C}$ in Fig. 5(a) and 6, the lower limit demixing enthalpy which is assumed as contributed by only the hydrogen bond effect also deviate significantly, that will introduce more errors in the estimate of entropic effect in the semidilute to dilute solution regions.

\section{Discussion}

The critical concentration when the sigmoidal demixing enthalpy recovery behavior occurs in PNIPAM aqueous solution is $49 \mathrm{wt} \%$, however, unlike the critical concentration of $38.3 \mathrm{wt} \%$ observed in aqueous PVME solution that corresponding to exactly $1: 2$ mole ratio between the repeating unit of PVME and water ${ }^{39}$ the physicochemical meaning of critical concentration in PNIPAM aqueous solution are poorly understood at present. These results can be compared with other experimental results in literature and help us to have a comprehensive understanding of the aqueous PNIPAM solution. For example, Ono et al. ${ }^{53}$ found that the hydration number of NIPAM monomers aqueous solution amounts to 5 , whereas that of linear PNIPAM chains in semidilute aqueous solutions is about 11-13. ${ }^{4,55}$ The simulation result showed that the maximum hydration number in $N$-methylacetamide/water system is $6,{ }^{56}$ in which $N$-methylacetamide has the same polar groups as the monomer of NIPAM. Accordingly, the concentration of water in aqueous solution with stoichiometric mole ratio of PNIPAM repeating unit and water as $1: 5$ is $44.33 \mathrm{wt} \%$, and for the mole ratio as $1: 6$ is $48.87 \mathrm{wt} \%$, which is very closed to the measured critical concentration of $49 \mathrm{wt} \%$ between region II and III in this study.

In extremely dilute good solvent-polymer solution, a polymer coil is in a swelling state, which is well investigated both theoretically ${ }^{57}$ and experimentally. ${ }^{58}$ While in the bulk melt state the polymer chain size is almost the same as that at the $\theta$ condition ${ }^{59}$ within experimental uncertainties. However, in the concentrated solution region, how and when the polymer coils begin to swell with increasing solvent concentration is still not well understood ${ }^{60}$ So far small-angle neutron scattering (SANS) method is the most powerful experimental technique to probe polymer coil size in concentrated polymer solutions. However, since the determination of the coil size of single polymer chain requires a mixture of deuterium labeled and common hydrogen polymer sample with approximately the same molecular weight and distribution, and the solution in zero average contrast neutron scattering condition. ${ }^{64}$ It goes without saying that these conditions are not easy to fulfill, and that is why the investigation of swelling of polymer coils in concentrated good solution has only been reported for polystyrene (PS) ${ }^{61-64}$ and poly(methyl methacrylate) (PMMA) ${ }^{65}$ in a few solvents in about fifty years since the development of SANS method. Moreover, due to the experimental uncertainty, the exact critical concentration when the polymer coil begins to swell is ambiguous and the estimated concentration value varies up to $10 \%$ or even higher; ${ }^{65}$ in turn, the physical meaning remains an open question. ${ }^{60}$ The DSC experiments conducted in PVME $^{39}$ and PNIPAM aqueous solutions in this investigation suggest that the entropic effect in the demixing enthalpy may be utilized as a promising alternative method to detect the conformational changes of polymer coils in concentrated solutions, although it can only obtain the relative changes, and at present these results can only be observed in LCST phase separation processes. However, a more precise critical concentration can be obtained from this method, which indicated that the mole ratio between the polymer repeating unit and solvent may play a critical role in the swelling of polymer coils. Based on the assumption, it is reasonable to interpret the phenomenon that polystyrene begins to swell in two different good solvents carbon disulfide ${ }^{61}$ and toluene $\mathrm{e}^{62-64}$ which are very different in volume concentration, but very close to $1: 1$ mole ratio for both solutions. SANS experiments in PNIPAM aqueous solutions have been reported in literatures, ${ }^{66,67}$ unfortunately, these works only conducted in hydrated PNIPAM and deuterated water mixture, which is much different from zero average neutron contrast condition, ${ }^{64}$ the information of the change of PVME coil size in concentrated aqueous solution could not be obtained. Our present DSC work would hopefully inspire more studies to investigate the microscopic characteristics using SANS or other techniques in concentrated aqueous PNIPAM solution in the future.

Ye $e t$ al. investigated the association of cyclic- and linearPNIPAM with varying chain lengths and end groups in dilute aqueous solutions ${ }^{68}$ and found the aggregation behaviors varies greatly. Moreover, the end-group or initiator in polymerization also influence the demixing behavior above LCST temperature. ${ }^{69,70}$ The results in this investigation showed that when water concentration is lower than $65 \mathrm{wt} \%$, the phase separation and demixing enthalpy recovery behavior in PNIPAM aqueous solutions were almost the same as in the previous study. ${ }^{41}$ However, when the water content is higher than $65 \mathrm{wt} \%$, the change of demixing enthalpy versus phase separation time at $55^{\circ} \mathrm{C}$ measured in this work is much different from our recent investigation. ${ }^{41}$ These results indicated that the influence of polymer chain architecture, chain lengths and end groups may affect the phase separation and aggregation in the semidilute and dilute aqueous PNIPAM solutions. Unfortunately, only the molecular weights and their distributions are known for the two samples, the polymerization procedure and end groups are not available. Moreover, due to the limitations in the heating and cooling scan rate of conventional DSC method, although a relative high cooling rate applied in this investigation, the macroscopic DSC experiment can only observe the demixing phenomenon in a long time scale. Although ultrafast DSC (the so-called flash DSC) can be utilized to investigate the influence of fast cooling and heating on the dynamic phenomena in homopolymer and polymer blends, ${ }^{71,72}$ but at 
this stage it can only be used to test solid samples. In contrast, laser temperature-jump time-resolved technique combined with other microscopic probing method can obtain the demixing information in a millisecond scale, ${ }^{73}$ which could help us in obtaining more comprehensive results on the aggregation behavior of polymer above LCST temperature.

\section{Conclusions}

In summary, the isothermal demixing enthalpy recovery behavior of aqueous PNIPAM solutions has been systematically investigated in this work. The variation of isothermal time exhibited distinct demixing enthalpy recovery behaviors in four concentration regions: (I) $0-24 \mathrm{wt} \%$, (II) $24-49 \mathrm{wt} \%$, (III) 49$65 \mathrm{wt} \%$, (IV) above $65 \mathrm{wt} \%$. In the case that the concentration of water is equal or lower than $24 \mathrm{wt} \%$, there is only a glass transition behavior in the DSC curve and no sign of phase separation can be observed for the mixtures. In region II, the isothermal demixing enthalpy recovery behavior possesses time independence. In concentration region III and IV, the further increasing water molecule plays a role of pure solvent to swell the polymer coils, resulting in the time dependence of phase separation enthalpy recovery behavior. Based on the dependence of demixing enthalpy recovery behavior on concentrations, the spring entropic effects in the phase separation of PNIAPM aqueous solution was quantitatively analyzed. The thermal effect contributed by spring entropy showed that at lower water concentrations of region II, the spring entropy is zero and it corresponds to compact globule state of PNIPAM coil. However, in region III, the spring entropy significantly increases with the increasing of water content, corresponding to the continuous swelling of the polymer coil as the increase of water concentration. Moreover, in region IV, the tendency of increasing in entropic effect with the raising of water concentration is weakened, indicating that the swelling degree of polymer coil reaches almost its maximum when the water concentration is $65 \mathrm{wt} \%$, and further increase in water concentration mainly induced dilution effect in the PNIPAM aqueous solution.

\section{Author contributions}

D. H. and S. J. conceived and designed the experiments. L. S., J. L. and P. L. planned and performed the DSC experiments. S. L., J. L. and P. L. analyzed the experimental data. L. S., J. L., P. L., J. L., S. J. and D. H. wrote the manuscript. All authors discussed the results, provided intellectual input and critical feedback and commented on the manuscript.

\section{Conflicts of interest}

There are no conflicts to declare.

\section{Acknowledgements}

This research was supported by the National Natural Science Foundation of China (Grant No. 20974079). The authors also would like to thank Yafei Huang at GE healthcare, Uppsala, Sweden for her kind help in polishing English of this manuscript.

\section{References}

1 K. Solc, K. Dusek, R. Koningsveld and H. Berghmans, "Zero" and "off-zero" critical concentrations in solutions of polydisperse polymers with very high molar masses, Collect. Czech. Chem. Commun., 1995, 60, 1661-1688.

2 A. Halperin, M. Kroger and F. M. Winnik, Poly $(N-$ isopropylacrylamide) phase diagrams: fifty years of research, Angew. Chem., Int. Ed., 2015, 54, 15342-15367.

3 Y. Okada and F. Tanaka, Cooperative Hydration, Chain Collapse, and Flat LCST Behavior in Aqueous $\operatorname{Poly}(\mathrm{N}$ isopropylacrylamide) Solutions, Macromolecules, 2005, 38, 4465-4471.

4 D. Schmaljohann, Thermo- and $\mathrm{pH}$-responsive polymers in drug delivery, Adv. Drug Delivery Rev., 2006, 58, 1655-1670.

5 A. S. Hoffman, Hydrogels for biomedical applications, $A d v$. Drug Delivery Rev., 2012, 64, 18-23.

6 S. Hocine and M. H. Li, Thermoresponsive self-assembled polymer colloids in water, Soft Matter, 2013, 9, 5839-5861.

7 T. Tanaka, I. Nishio, S. T. Sun and S. Uenonishio, Collapse of gels in an electric field, Science, 1982, 218, 467-469.

8 A. Mamada, T. Tanaka, D. Kungwatchakun and M. Irie, Photoinduced phase transition of gels, Macromolecules, 1990, 23, 1517-1519.

9 A. Zhuk, R. Mirza and S. Sukhishvili, Multiresponsive claycontaining layer-by-layer films, ACS Nano, 2011, 5, 87908799.

10 Y. Guan, H. B. Zhao, L. X. Yu, S. C. Chen and Y. Z. Wang, Multi-stimuli sensitive supramolecular hydrogel formed by host-guest interaction between PNIPAM-Azo and cyclodextrin dimers, RSC Adv., 2014, 4, 4955-4959.

11 L. Wang, L. Liu, B. Y. Dong, H. Y. Zhao, M. M. Zhang, W. J. Chen and Y. H. Hong, Multi-stimuli-responsive biohybrid nanoparticles with cross-linked albumin coronae self-assembled by a polymer-protein biodynamer, Acta Biomater., 2017, 54, 259-270.

12 F. L. Wang, M. A. Wang, D. Song, Y. Xu and D. H. Huang, Synthesis of water soluble block copolymer with temperature and chemical dual-stimuli responsive and its application in renewable heavy metal ion adsorbent, $J$. Polym. Mater., 2017, 34, 615-630.

13 H. H. Li, J. P. O'Shea and G. V. Franks, Effect of molecular weight of poly( $N$-isopropyl acrylamide) temperaturesensitive flocculants on dewatering, AIChE J., 2009, 55, 2070-2080.

14 H. Kanazawa, M. Nishikawa, A. Mizutani, C. Sakamoto, Y. Morita-Murase, Y. Nagata, A. Kikuchi and T. Okano, Aqueous chromatographic system for separation of biomolecules using thermoresponsive polymer modified stationary phase, J. Chromatogr. A, 2008, 1191, 157-161.

15 K. N. Plunkett, X. Zhu, J. S. Moore and D. E. Leckband, PNIPAM Chain Collapse Depends on the Molecular Weight and Grafting Density, Langmuir, 2006, 22, 4259-4266. 
16 H. Yim, M. S. Kent, S. Mendez, G. P. Lopez, S. Satija and Y. Seo, Temperature-dependent conformational changes of PNIPAM grafted chains in water: effects of molecular weight and grafting density, Macromolecules, 2006, 39, 3420-3426.

17 K. Wu, B. Wu, P. Wang, Y. Hou, G. Z. Zhang and D. M. Zhu, Adsorption Isotherms and Dissipation of Adsorbed Poly $(\mathrm{N}$ isopropylacrylamide) in Its Swelling and Collapsed States, J. Phys. Chem. B, 2007, 111, 8723-8727.

18 D. M. Zhu, K. Wu, B. Wu, P. Wang, J. J. Fang, Y. Hou and G. Z. Zhang, Physisorption of Poly( $N$-isopropylacrylamide) in Its Swollen and Collapsed States: Effects of Molecular Conformation and Substrate Interaction, J. Phys. Chem. C, 2007, 111, 18679-18686.

19 X. Laloyaux, B. Mathy, B. Nysten and A. M. Jonas, Surface and Bulk Collapse Transitions of Thermoresponsive Polymer Brushes, Langmuir, 2010, 26, 838-847.

20 C. Y. Xue, N. Yonet-Tanyeri, N. Brouette, M. Sferrazza, P. V. Braun and D. E. Leckband, Protein adsorption on poly( $N$-isopropylacrylamide) brushes: dependence on grafting density and chain collapse, Langmuir, 2011, 27, 8810-8818.

21 E. S. Kooij, X. F. Sui, M. A. Hempenius, H. J. W. Zandvliet and G. J. Vancso, Probing the Thermal Collapse of Poly $(\mathrm{N}$ isopropylacrylamide) Grafts by Quantitative In situ Ellipsometry, J. Phys. Chem. B, 2012, 116, 9261-9268.

22 O. B. Ptitsyn and I. E. Eizner, The theory of helix-coil transitions in macromolecules, Biofizika, 1965, 10, 3-6.

23 P. G. de Gennes, Exponents for the excluded volume problem as derived by the Wilson method, Phys. Lett. A, 1972, 38, 339-340.

24 P. G. de Gennes, Collapse of a polymer chain in poor solvents, J. Phys., Lett., 1975, 36, 55-57.

25 I. Nishio, S. T. Sun, G. Swislow and T. Tanaka, First observation of the coil-globule transition in a single polymer chain, Nature, 1979, 281, 208-209.

26 I. H. Park, Q. W. Wang and B. Chu, Transition of linear polymer dimensions from THETA to collapsed regime. 1. Polystyrene/cyclohexane system, Macromolecules, 1987, 20, 1965-1975.

27 M. Nakata and T. Nakagawa, Kinetics of coil-globule transition of poly(methyl methacrylate) in isoamyl acetate, J. Chem. Phys., 1999, 110, 2703-2710.

28 T. Terramoto and F. Yonezawa, Coil-globule transition of a semi-flexible chain, Int. J. Mod. Phys. B, 2000, 14, 621-633.

29 L. L. He, G. Cheng and Y. B. Melnichenko, Partial Collapse and Reswelling of a Polymer in the Critical Demixing Region of Good Solvents, Phys. Rev. Lett., 2012, 109, 067801.

30 P. Thiyagarajan, D. J. Chaiko and R. P. Hjelm, A Neutron Scattering Study of Poly(ethylene glycol) in Electrolyte Solutions, Macromolecules, 1995, 28, 7730-7736.

31 P. A. Gurnev, C. B. Stanley, M. A. Aksoyoglu, K. L. Hong, V. A. Parsegian and S. M. Bezrukov, Poly(ethylene glycol)s in Semidilute Regime: Radius of Gyration in the Bulk and Partitioning into a Nanopore, Macromolecules, 2017, 50, 2477-2483.
32 R. M. Neuman, Nonequivalence of the stress and strain ensembles in describing polymer-chain elasticity, Phys. Rev. A, 1985, 31, 3516-3517.

33 R. M. Neuman, Implications of using the entropy spring model for an ideal polymer chain, Phys. Rev. A, 1986, 34, 3487-3488.

34 T. M. Birshtein and V. A. Pryamitsyn, Coil-globule type transitions in polymers. 2 . Theory of coil-globule transition in linear macromolecules, Macromolecules, 1991, 24, 15541560.

35 M. D. Wang, H. Yin, R. Landick, J. Gelles and S. M. Block, Stretching DNA with optical tweezers, Biophys. J., 1997, 72, 1335-1346.

$36 \mathrm{~W}$. Zhang and X. Zhang, Single molecule mechanochemistry of macromolecules, Prog. Polym. Sci., 2003, 28, 1271-1295.

37 C. Liu, W. Shi, S. Cui, Z. Wang and X. Zhang, Force spectroscopy of polymers: Beyond single chain mechanics, Curr. Opin. Solid State Mater. Sci., 2005, 9, 140-148.

38 N. M. Toan and D. Thirumalai, On the origin of the unusual behavior in the stretching of single-stranded DNA, J. Chem. Phys., 2012, 136, 235103.

39 L. Song, J. X. Lin, Y. He, J. Q. Li, J. Sheng, S. C. Jiang and D. H. Huang, Entropic effect implication for change in polymer coils swelling state in the demixing enthalpy recovery of aqueous poly(vinyl methyl ether) solutions, $J$. Polym. Sci., Part B: Polym. Phys., 2019, 57, 142-151.

40 Y. A. Budkov, A. L. kolesnikov, N. Georgi and M. G. Kiselev, A flexible polymer chain in a critical solvent: coil or globule?, Europhys. Lett., 2015, 109, 36005.

41 P. P. Liu, L. Song, N. C. Li, J. X. Lin and D. H. Huang, Time dependence of phase separation enthalpy recovery behavior in aqueous poly( $N$-isopropyl acrylamide) solutions, J. Therm. Anal. Calorim., 2017, 130, 843-850.

42 L. Song, J. X. Lin, Y. He, J. Q. Li, J. Sheng, S. C. Jiang and D. H. Huang, Dual entropic and enthalpic processes in the lower critical solution temperature phase separation of poly(vinyl methyl ether) aqueous solutions, J. Polym. Sci., Part B: Polym. Phys., 2019, 57, 323-330.

43 L. Liu and Q. X. Guo, Isokinetic relationship, isoequilibrium relationship, and enthalpy-entropy compensation, Chem. Rev., 2001, 101, 673-696.

44 A. Pan, T. Biswas, A. K. Rakshit and S. P. Moulik, Enthalpyentropy compensation (EEC) effect: a revisit, J. Phys. Chem. B, 2015, 119, 15876-15884.

45 K. K. Frederick, M. S. Marlow, K. G. Valentine and A. J. Wand, Conformation entropy in molecular recognition by proteins, Nature, 2007, 448, 325-329.

46 S. R. Tzeng and C. G. Kalogimos, Protein activity regulation by conformational entropy, Nature, 2012, 488, 236-240.

47 J. A. Caro, K. W. Harpole, V. Kasinath, J. Lim, J. Granja, K. G. Valentine, K. A. Sharp and A. J. Wand, Entropy in molecular recognition by proteins, Proc. Natl. Acad. Sci. U. S. A., 2017, 114, 6563-6568.

48 T. Ube, H. Aoki, S. Ito, J. Horinaka and T. Takigawa, Relaxation of single polymer chain in binary molecular weight blends observed by scanning near-field optical microscopy, Soft Matter, 2012, 8, 5603-5611. 
49 T. P. Lodge and M. Muthukumar, Physical chemistry of polymers: entropy, interactions, and dynamics, J. Phys. Chem., 1996, 100, 13275-13292.

50 C. Wu and X. H. Wang, Globule-to-coil transition of a single homopolymer chain in solution, Phys. Rev. Lett., 1998, 80, 4092-4094.

51 F. Afroze, E. Nies and H. Berghmans, Phase transitions in the system poly( $N$-isopropylacrylamide)/water and swelling behaviour of the corresponding networks, J. Mol. Struct., 2000, 554, 55-68.

52 Y. W. Ding, X. D. Ye and G. Z. Zhang, Microcalorimetric investigation on aggregation and dissolution of $\operatorname{poly}(\mathrm{N}$ isopropylacrylamide) chains in water, Macromolecules, 2005, 38, 904-908.

53 Y. Ono and T. Shikata, Contrary hydration behavior of $N$ isopropylacrylamide to its polymer, P(NIPAm), with a lower critical solution temperature, J. Phys. Chem. B, 2007, 111, 1511-1513.

54 Y. Ono and T. Shikata, Hydration and dynamic behavior of poly( $N$-isopropylacrylamide)s in aqueous solution: a sharp phase transition at the lower critical solution temperature, J. Am. Chem. Soc., 2006, 128, 10030-10031.

55 Y. Satokawa, T. Shikata, F. Tanaka, X. P. Qiu and F. M. Winnik, Hydration and dynamic behavior of a cyclic poly( $N$-isopropylacrylamide) in aqueous solution: effects of the polymer chain topology, Macromolecules, 2009, 42, 1400-1403.

56 Z. Z. Yang and P. A Qian, Study of $N$-methylacetamide in water clusters: based on atom-bond electronegativity equalization method fused into molecular mechanics, $J$. Chem. Phys., 2006, 125, 064311.

57 M. Daoud and G. Jannink, Temperature-concentration diagram of polymer solutions, J. Phys., 1976, 37, 973-979.

58 D. W. Shaefer and C. C. Han, in Dynamic Light Scattering., ed. R. Pecora, Plenum, New York, 1985.

59 W. W. Graessley, Polymeric Liquids and Networks: Structure and Properties, Garland Books, New York, 2004.

60 S. Westermann, L. Willner, D. Richter and L. J. Fetters, The evaluation of polyethylene chain dimensions as a function of concentration in nonadecane, Macromol. Chem. Phys., 2000, 201, 500-504.

61 M. Daoud, J. P. Cotton, B. Farnoux, G. Jannik, G. Sarma, H. Benoit, R. Duplessix, C. Picot and P. G. de Gennes, Solutions of Flexible Polymers. Neutron Experiments and Interpretation, Macromolecules, 1975, 8, 804-818.

62 J. S. King, W. Boyer, G. D. Wignall and R. Ullmam, Radii of gyration and screening lengths of polystyrene in toluene as a function of concentration, Macromolecules, 1985, 18, 709718.
63 W. Brown, K. Mortensen and G. Floudas, Screening lengths in concentrated polystyrene solutions in toluene determined using small-angle neutron and small angle X-ray scattering, Macromolecules, 1992, 25, 6904-6908.

64 G. D. Wignall and Y. B. Melnichenko, Recent applications of small-angle neutron scattering in strongly interacting soft condensed matter, Rep. Prog. Phys., 2005, 68, 1761-1804.

65 G. Cheng, W. W. Graessley and Y. B. Melnichenko, Polymer Dimensions in Good Solvents: Crossover from Semidilute to Concentrated Solutions, Phys. Rev. Lett., 2009, 102, 157801.

66 A. Meier-Koll, V. Pipich, P. Busch, C. M. Papadakis and P. Muller-Buschbaum, Phase Separation in Semidilute Aqueous Poly( $N$-isopropylacrylamide) Solutions, Langmuir, 2012, 28, 8791-8798.

67 K. Nishi, T. Hiroi, K. Hashimoto, K. Fujii, Y. S. Han, T. H. Kim, Y. Katsumoto and M. Shibayama, SANS and DLS Study of Tacticity Effects on Hydrophobicity and Phase Separation of Poly( $N$-isopropylacrylamide), Macromolecules, 2013, 46, 6225-6232.

68 J. Ye, J. Xu, J. Hu, X. Wang, G. Zhang, S. Liu and C. Wu, Comparative Study of Temperature-Induced Association of Cyclic and Linear Poly( $N$-isopropylacrylamide) Chains in Dilute Solutions by Laser Light Scattering and StoppedFlow Temperature Jump, Macromolecules, 2008, 41, 44164422 .

69 K. Otake, H. Inomata, M. Konno and S. Saito, Thermal analysis of the volume phase transition with $\mathrm{N}$ isopropylacrylamide gels, Macromolecules, 1990, 23, 283289.

70 K. Chan, R. Pelton and J. Zhang, On the Formation of Colloidally Dispersed Phase-Separated Poly $(\mathrm{N}$ isopropylacrylamide), Langmuir, 1999, 15, 4018-4020.

71 S. C. Luo, L. Wei, J. Jiang, Y. Sha, G. Xue, X. L. Wang and D. S. Zhou, Phase separation dynamics of a poly(vinyl methyl ether)/polystyrene (PVME/PS) blend studied by ultrafast differential scanning calorimetry, J. Polym. Sci., Part B: Polym. Phys., 2017, 55, 1357-1364.

72 S. C. Luo, X. Kui, E. R. Xing, X. L. Wang, G. Xue, C. Schick, W. B. Hu, E. Zhuravkev and D. S. Zhou, Interplay between free surface and solid interface nucleation on two-step crystallization of poly(ethylene terephthalate) thin films studied by fast scanning calorimetry, Macromolecules, 2018, 51, 5209-5218.

73 D. Kato, W. Y. Sohn and K. Katayama, Aggregation-Induced Expansion of Poly-( $N$-isopropyl acrylamide) Solutions Observed Directly by the Transient Grating Imaging Technique, ACS Omega, 2018, 3, 8484-8490. 